\title{
On the Links Between Managerial Actions, Keystone Species, and the Resilience of Ecological-Economic Systems ${ }^{1}$
}

by

\author{
Amitrajeet A. Batabyal ${ }^{2}$
}

This paper is dedicated to the memory of A.N. Batabyal (1937-2002). We acknowledge financial support from the Gosnell endowment at RIT. The usual disclaimer applies.

2

Department of Economics, Rochester Institute of Technology, 92 Lomb Memorial Drive, Rochester, NY 14623-5604, USA. Internet aabgsh@rit.edu 


\title{
On the Links Between Managerial Actions, Keystone Species, and the Resilience of Ecological-Economic Systems
}

\begin{abstract}
Managerial actions influence the survival probabilities of the keystone species of an ecological-economic system. In turn, the well-being of these keystone species translates into the wellbeing or the resilience of the underlying ecological-economic system. What are the theoretical links between managerial actions, keystone species, and the resilience of ecological-economic systems? Recently, Batabyal (2002) has addressed aspects of this question. In this paper, we extend the analysis in Batabyal (2002). First, we use a stochastic model to delineate a stylized ecological-economic system. Next, we discuss the links between managerial actions, keystone species, and this ecologicaleconomic system's resilience. Finally, we show how the model can be used to actually compute the resilience of our ecological-economic system.
\end{abstract}

Keywords: Ecological-Economic System, Keystone Species, Markov Chain, Resilience, Uncertainty JEL Classification: Q20, D80, D81 


\section{On the Links Between Managerial Actions, Keystone Species,}

\section{and the Resilience of Ecological-Economic Systems}

\section{Introduction}

Within a specific ecological-economic system, each species is generally linked to and also depends on other species. In addition, each species also contributes to the overall unity of this ecological-economic system. Even so, although all species contribute to the functioning of an ecological-economic system, in the grand scheme of things, some species clearly do more than others. Specifically, some species render key services that are also unique. Without the work of these species, the underlying ecological-economic system would be very different. Ecologists call such fundamental players keystone species. ${ }^{3}$

When a keystone species vanishes from an ecological-economic system, this system changes spectacularly. In fact, what is particularly salient is that the keystone's disappearance often activates the loss of other resident species, and when this happens, the complex connections among the remaining resident species begin to unravel. In the words of Batabyal (2002, p. 3), as "the loss of one species prompts the loss of still other species, in a veritable domino effect, species losses cascade through the ecological-economic system."

This discussion tells us that ensuring the survival of keystone species ought to be an essential part of the task of managing ecological-economic systems efficaciously. The significance of this link between the survival of keystone species and the well-being of ecological-economic systems has been

A standard textbook in ecology tells us that a "role may be occupied by a single species, and the presence of that role may be critical to the community. Such important species are called keystone species because their activities determine community structure" (Krebs, 1994, p. 554, italics in original). 
commented on by many researchers. For example, Simberloff (1998, p. 247) has noted that if "we can identify keystone species and the mechanisms that cause them to have such wide-ranging impacts, we would almost certainly derive information on the functioning of the entire [ecological-economic system] that would be useful in its management."

The above citation tells us that there is a link between managerial actions and the survival of keystone species. Now, it should be clear to the reader that an ably managed ecological-economic system will provide humans with a whole host of consumptive and non-consumptive services. Using the language of ecological stability theory, we would say that such an ably managed system is resilient, where resilience refers to "the amount of disturbance that can be sustained [by an ecological-economic system] before a change in system control or structure occurs" (Holling et al., 1995, p. 50). ${ }^{4}$ There are many ways to quantify the resilience of an ecological-economic system. One prominent recent line of research tells us that the resilience of an ecological-economic system is appropriately viewed as the steady state probability of being in one or more desirable states of the system. ${ }^{5}$ This is also the interpretation of resilience that we shall use in this paper.

Thus far, we have seen that managerial actions affect the survival probabilities and hence the well-being of keystone species. In turn, the well-being of keystone species translates into the wellbeing or the resilience of the underlying ecological-economic system. Put differently, we have now verbally demonstrated a link between the trio of managerial actions, the survival of keystone species,

The term resilience has two meanings in the ecology literature. The first meaning, due to Pimm (1984), concentrates on stability near an equilibrium and stresses the speed of return to this equilibrium. In contrast, the second meaning (and the meaning we're using in this paper) is due to Holling (1973) and it concentrates on conditions far from an equilibrium where perturbations can flip a system into an alternate stability domain.

5

See Perrings (1998), Batabyal (2000, 2002), and Batabyal and Beladi (1999) for more on this research. 
and the resilience of ecological-economic systems. However, what are the theoretical links between this trio? Recently, Batabyal (2002) has addressed aspects of this question. Specifically, Batabyal (2002) uses a discrete-time Markov chain theoretic model of an ecological-economic system to shed light on the theoretical links between the above discussed trio. Although Batabyal's (2002) paper does advance the extant literature, the analysis in this paper is confining in three ways. First, there are only two states in the model. Second, Batabyal conducts his analysis with a single keystone species. Finally, the link between managerial actions and keystone species survival is very specific.

As such, in this paper we dispense with all three of these confining features and thereby extend the analysis in Batabyal (2002). Specifically, we analyze a five state discrete-time Markov chain theoretic model in which the states are defined in a way so as to clearly bring out the general link between managerial actions and keystone species survival. Second, in our ecological-economic system, there are two keystone species and we explicitly model-to the best of our knowledge for the first time - an important kind of interaction between the two keystone species in this ecologicaleconomic system.

The rest of this paper is organized as follows. Section 2.1 delineates our discrete-time Markov chain theoretic model ${ }^{6}$ of an ecological-economic system with two keystone species. Section 2.2 demonstrates how to construct the transition probability matrix for this system and then calculates the resilience of this system. Section 2.3 discusses the findings of the previous two sections. Section 3 concludes and suggests avenues for future research on the subject of this paper.

Taylor and Karlin (1998) and Ross (2000) contain textbook accounts of discrete-time Markov chains. 


\section{A Stochastic Model of an Ecological-Economic System}

\subsection{Preliminaries}

Consider an arbitrary ecological-economic system with two keystone species. Examples of such systems include temperate bogs in which the peat forming mosses Polytrichum strictum and Sphagnum fallax are the keystone species (Mitchell et al. (2002)) and some Amazonian forests in which the gum producing tree species Parkia nitida and Parkia pendula are considered to be keystone species (Peres (2000)). These examples notwithstanding, the best example of an ecologicaleconomic system with two keystone species is probably the North American mixed grass prairie in which both bisons and prairie dogs are considered to be keystone species (Fahnestock and Detling (2002)).

We now abstract away from the remaining species of our ecological-economic system and we concentrate on the two keystone species. ${ }^{7}$ Hence, in our model, the well-being of the ecologicaleconomic system under study depends essentially on the well-being of these two keystone species. As such, we shall say that our ecological-economic system is functional ${ }^{8}$ if and only if at least one of the two keystone species is healthy and not endangered. Now, economic activities such as fishing, grazing, and hunting, and uncertain environmental occurrences such as droughts, fires, and winter freezes, influence the health of the two keystone species and hence the health of our ecologicaleconomic system. Naturally, inordinate levels of such economic activities and/or unusually extreme environmental occurrences can endanger the lives of these keystone species. Therefore, when the

7

This approach has been used previously in the literature by Batabyal (2000, 2002) and Batabyal and Beladi (1999).

8

In the sense that this ecological-economic system is able to provide humans with key ecological services such as flood protection, nutrient cycling, and the maintenance of the hydrological cycle. 
manager of our ecological-economic system suspects that one or both keystone species are endangered, (s)he takes apposite preventive actions to ensure that these keystone species do not become extinct and thereby imperil the entire system. Examples of such actions include season length restrictions on fishing, a moratorium on grazing, and a ban on hunting.

Now, consider an arbitrary time period. Suppose that at the beginning of this time period, the two keystone species in our ecological-economic system are healthy. Then, because of the activities mentioned in the previous paragraph, during this time period, each of the two keystone species will be endangered-and hence our ecological-economic system will be endangered-with probability $p$. To capture the interaction between these two keystone species, ${ }^{9}$ we suppose that when one keystone species is endangered in a particular time period, the second keystone species will also be endangered with a higher probability $q$. That is, $q>p$. As indicated in the previous paragraph, when one or both keystone species are endangered, our manager takes the necessary prophylactic actions. Clearly, the aim of actions such as a ban on hunting is to "nurse" the affected keystone species back to health. In this paper, we suppose that the manager's expertise is such that the "nursing period" is two time periods long. ${ }^{10}$ We now formally model the behavior of our stylized ecological-economic system as a discrete-time Markov chain.

\subsection{Analysis}

The first step in this modeling procedure involves the specification of an appropriate set of states. To this end, let $x$ denote the number of keystone species that are healthy (not endangered) and

9

Such as the interaction between the bison and the prairie dog in North American mixed grass prairies.

10

As an alternative, we could let the length of this "nursing period" be a random variable. For more on this alternative, see section 3. 
let $y$ denote the time spent by one or both keystone species being "nursed" back to health by the manager of our ecological-economic system. The reader will note that the possible numerical values of $x$ are 0,1 , and 2 , and similarly, the possible numerical values of $y$ are 0 and 1 .

Given this information, let us represent the state of our ecological-economic system by the pair $(x, y)$. Then, we have a five state, discrete-time Markov chain theoretic model of our ecologicaleconomic system. Specifically, the five states are $(2,0),(1,0),(1,1),(0,0)$, and $(0,1)$. Our next task is to specify the twenty-five one-step transition probabilities. This task is simplified by the fact that many of these one-step transition probabilities are either one or zero. For instance, the probability of making a transition from state $(0,0)$ to state $(0,1)$ is one. Similarly, the probability of making a transition from state $(2,0)$ to state $(1,1)$ is zero.

Naturally, some transition probabilities are neither one nor zero. Here are two examples. First, consider the probability of making a transition from state $(2,0)$ to state $(2,0)$. Now, in any particular time period, the probability that a keystone species is healthy is $(1-p)$. Our ecological-economic system contains two keystone species. Putting these two pieces of information together, we reason that the probability of making a transition from state $(2,0)$ to state $(2,0)$ is $(1-p)^{2}$. Second, consider the probability of making a transition from state $(1,0)$ to state $(1,1)$. Recall that in any time period, $q>p$ is the probability that the second keystone species is endangered, given that the first keystone species is endangered. Further, the value of $x$ is unchanged and the value of $y$ increases from 0 to 1 . These two pieces of information together tell us that the relevant transition probability that we seek is $(1-q)$. The values of the other one-step transition probabilities that are neither one nor zero can be obtained by similar reasoning. Therefore, the one-step transition probability matrix for our five state ecological-economic system is 


$$
P=\left[\begin{array}{lllll}
P_{(2,0)(2,0)} & P_{(2,0)(1,0)} & P_{(2,0)(1,1)} & P_{(2,0)(0,0)} & P_{(2,0)(0,1)} \\
P_{(1,0)(2,0)} & P_{(1,0)(1,0)} & P_{(1,0)(1,1)} & P_{(1,0)(0,0)} & P_{(1,0)(0,1)} \\
P_{(1,1)(2,0)} & P_{(1,1)(1,0)} & P_{(1,1)(1,1)} & P_{(1,1)(0,0)} & P_{(1,1)(0,1)} \\
P_{(0,0)(2,0)} & P_{(0,0)(1,0)} & P_{(0,0)(1,1)} & P_{(0,0)(0,0)} & P_{(0,0)(0,1)} \\
P_{(0,1)(2,0)} & P_{(0,1)(1,0)} & P_{(0,1)(1,1)} & P_{(0,1)(0,0)} & P_{(0,1)(0,1)}
\end{array}\right]=\left[\begin{array}{ccccc}
(1-p)^{2} & 2 p(1-p) & 0 & p^{2} & 0 \\
0 & 0 & (1-q) & 0 & q \\
(1-q) & q & 0 & 0 & 0 \\
0 & 0 & 0 & 0 & 1 \\
0 & 1 & 0 & 0 & 0
\end{array}\right] .
$$

This completes the probabilistic description of our stylized ecological-economic system. So far, we have seen that the system under study can be well described by a five state, discrete-time Markov chain, whose transition probability matrix $P$ is given by equation (1).

We now want to theoretically link the trio of managerial actions, the survival of the two keystone species, and our ecological-economic system's resilience. To this end, note that managerial actions arise in states $(1,0),(1,1),(0,0)$ and state $(0,1)$. In these four states, at least one keystone species is endangered and prophylactic actions are taken by our manager. Further, the two time periods it takes to "nurse" an endangered keystone species back to health affect the survival probabilities or alternately the health of the keystone species. Examples of such survival probabilities include $P_{(1,1),(2,0)}=(1-q)$ and $P_{(1,0),(1,1)}=(1-q)$. This is the first link- between managerial actions and the survival of the keystone species_-in the above mentioned trinity.

Recall from section 1 that the resilience of our ecological-economic system is defined to be the steady state probability of being in one or more desirable states of this system. How many desirable states does our ecological-economic system have? A strict interpretation of the above definition tells us that the only desirable state is $(2,0)$. In this state, both the keystone species are healthy and hence preventive actions on the part of the manager are not needed. Hence, following this strict interpretation, resilience is the steady state probability of being in state $(2,0)$. A weak interpretation of the definition of resilience would involve equating resilience with the steady state 
probability that our ecological-economic system is functional. This system is functional when at least one of the two keystone species is healthy and this happens in states $(2,0),(1,0)$, and $(1,1)$. Therefore, following this weak interpretation, resilience is the sum of the steady state probabilities of being in states $(2,0),(1,0)$, and $(1,1)$. This discussion tells us that there are two ways of conceptualizing the desirable states of our ecological-economic system. Consequently, irrespective of whether we use the strict or the weak interpretation of the definition of resilience, once we've computed the resilience of our stylized ecological-economic system, we will have theoretically ascertained the second and final link in the above mentioned trio.

As noted in Taylor and Karlin (1998, p. 199), in terms of the transition probabilities in equation (1), given any initial state $i$, the steady state probability of being in state $j$ is

$$
\lim _{\{t \rightarrow \infty\}} P_{i j}^{t}=\pi_{j}, j=(2,0),(1,0),(1,1),(0,0),(0,1) .
$$

Following the strict interpretation, our ecological-economic system's resilience is $\pi_{(2,0)}$. Similarly, following the weak interpretation, this system's resilience is $\pi_{(2,0)}+\pi_{(1,0)}+\pi_{(1,1)}$. Using Theorem 1.1 in Taylor and Karlin (1998, p. 204), the actual values of these steady state probabilities can be computed. The essential idea here is to solve a system of five equations in the five unknown steady state probabilities. Although the relevant computations are straightforward, they are very tedious. Consequently, to illustrate our methodology, in the remainder of this section we suppose that $p=0.1$ and $q=0.2$. Now, using these values for $p$ and $q$ along with Theorem 1.1 in Taylor and Karlin (1998, p. 204), it is easy to see that $\pi_{(2,0)}=0.6197, \pi_{(1,0)}=0.1840$, and $\pi_{(1,1)}=0.1472$. Therefore, we find that for our ecological-economic system,

Strict Resilience $=\pi_{(2,0)}=0.6197$ and Weak Resilience $=\pi_{(2,0)}+\pi_{(1,0)}+\pi_{(1,1)}=0.9508$.

Equation (3) gives us the resilience of our ecological-economic system and the second 
link—-between the survival of the keystone species and the system's resilience- that we're after. Intuitively, we expect the relation $0 \leq$ Strict Resilience $\leq$ Weak Resilience $\leq 1$ to hold generally. In the illustrative case discussed in the previous paragraph, we see that $0 \leq 0.6197 \leq 0.9508 \leq 1$ and hence the above relation does indeed hold.

\subsection{Discussion}

Our analysis of the trio of managerial actions, keystone species survival, and the resilience of ecological-economic systems yields four noteworthy conclusions. First, given the uncertain nature of economic activities and environmental occurrences, an examination of the above mentioned trio calls for the analysis of a stochastic model. Second, the survival of the two keystone species of our ecological-economic system is contingent on the nature of the managerial actions taken when one or more such species are endangered. Third, by altering the kind and the level of these actions, the manager can directly influence the well-being or the resilience of the underlying ecological-economic system. Finally, the links between managerial actions, keystone species survival, and the resilience of ecological-economic systems can be quantified in the way that we have shown in this paper.

\section{Conclusions}

In this paper, we used a five state, discrete-time Markov chain theoretic model and provided a novel illustration of the theoretical links between managerial actions, the survival of keystone species, and the resilience of a stylized ecological-economic system. Our principal findings are described in section 2.3. The analysis of this paper can be extended in a number of different directions. In what follows, we propose two potential extensions.

First, we analyzed a model in which the amount of time it takes our manager to "nurse" a keystone species back to health is deterministic (two time periods long). However, as noted in 
footnote 10, one way to extend the analysis of this paper would be to allow the length of this "nursing period" to be stochastic. The resulting model with this feature would be richer and this richer model would, most likely, shed additional light on the links that we've analyzed in this paper. Second, we modeled the interaction between the two keystone species of our ecological-economic system in a very specific way. Although we believe this to be a useful start, it would nonetheless be helpful to consider more general ways of modeling this interaction, perhaps using the theory of associated random variables as discussed in Ross (1996). Studies that integrate these facets of the problem into the analysis will broaden our knowledge of the links between managerial actions, keystone species survival, and the resilience of ecological-economic systems. 


\section{References}

Batabyal, A.A. 2000. An Analysis of Persistence, Resilience, and the Conservation of Keystone Species, International Journal of Ecology and Environmental Sciences, 26, 1-10.

Batabyal, A.A. 2002. Human Actions, the Survival of Keystone Species, and the Resilience of Ecological-Economic Systems. Forthcoming, Resources Policy.

Batabyal, A.A., and Beladi, H. 1999. The Stability of Stochastic Systems: The Case of Persistence and Resilience, Mathematical and Computer Modelling, 30, 27-34.

Fahnestock, J.T., and Detling, J.K. 2002. Bison-Prairie Dog-Plant Interactions in a North American Mixed-Grass Prairie, Oecologia, 132, 86-95.

Holling, C.S. 1973. Resilience and Stability of Ecological Systems, Annual Review of Ecology and Systematics, 4, 1-23.

Holling, C.S., Schindler, D.W., Walker, B.W., and Roughgarden, J. 1995. Biodiversity in the Functioning of Ecosystems: An Ecological Synthesis, in C. Perrings, K.G. Maler, C. Folke, C.S. Holling, and B.O. Jansson, (Eds.), Biodiversity Loss. Cambridge University Press, Cambridge, United Kingdom.

Krebs, C.J. 1994. Ecology, $4^{\text {th }}$ edition. Addison Wesley Longman, Menlo Park, California.

Mitchell, E.A.D., Buttler, A., Grosvernier, P., Rydin, H., Siegenthaler, A., and Gobat, J. 2002. Contrasted Effects of Increased $\mathrm{N}$ and $\mathrm{CO}_{2}$ Supply on Two Keystone Species in Peatland Restoration and Implications for Global Change, Journal of Ecology, 90, 529-533.

Peres, C.A. 2000. Identifying Keystone Plant Resources in Tropical Forests: The Case of Gums from Parkia Pods, Journal of Tropical Ecology, 16, 287-317.

Perrings, C. 1998. Resilience in the Dynamics of Economy-Environment Systems, Environmental 
and Resource Economics, 11, 503-520.

Pimm, S.L. 1984. The Complexity and Stability of Ecosystems, Nature, 307, 321-326.

Ross, S.M. 1996. Stochastic Processes, $2^{\text {nd }}$ edition. Wiley, New York, New York.

Ross, S.M. 2000. Introduction to Probability Models, $7^{\text {th }}$ edition. Harcourt Academic Press, San Diego, California.

Simberloff, D. 1998. Flagships, Umbrellas, and Keystones: Is Single-Species Management Passe in the Landscape Era? Biological Conservation, 83, 247-257.

Taylor, H.M., and Karlin, S. 1998. An Introduction to Stochastic Modeling, $3^{\text {rd }}$ edition. Academic Press, San Diego, California. 